\title{
Human-Robot Similarity and Willingness to Work with a Robotic Co-worker
}

\author{
Sangseok You \\ 105 Hinds Hall \\ Syracuse, NY 13244 USA \\ Syracuse University \\ syou03@syr.edu
}

\author{
Lionel P. Robert Jr. \\ 105 S. State St. \\ Ann Arbor, MI 48109 USA \\ University of Michigan \\ lprobert@umich.edu
}

\begin{abstract}
Organizations now face a new challenge of encouraging their employees to work alongside robots. In this paper, we address this problem by investigating the impacts of human-robot similarity, trust in a robot, and the risk of physical danger on individuals' willingness to work with a robot and their willingness to work with a robot over a human co-worker. We report the results from an online experimental study involving 200 participants. Results showed that human-robot similarity promoted trust in a robot, which led to willingness to work with robots and ultimately willingness to work with a robot over a human co-worker. However, the risk of danger moderated not only the positive link between the surface-level similarity and trust in a robot, but also the link between intention to work with the robot and willingness to work with a robot over a human coworker. We discuss several implications for the theory of human-robot interaction and design of robots.
\end{abstract}

\section{CCS CONCEPTS}

- Human-centered computing $\rightarrow$ Human-computer interaction (HCI)

\section{KEYWORDS}

Human-robot teamwork, robot adoption, intention to work with robots, trust, similarity, deep-level, surface-level

\section{INTRODUCTION}

Robots are increasingly being adopted to both replace and work alongside humans [72]. Robots are being employed across assembly lines, order fulfillment centers, and product inspections service centers [32]. For instance, Amazon is adding 15,000

Permission to make digital or hard copies of all or part of this work for personal or classroom use is granted without fee provided that copies are not made or distributed for profit or commercial advantage and that copies bear this notice and the full citation on the first page. Copyrights for components of this work owned by others than the author(s) must be honored. Abstracting with credit is permitted. To copy otherwise, or republish, to post on servers or to redistribute to lists, requires prior specific permission and/or a fee. Request permissions from Permissions@acm.org.

HRI '18, March 5-8, 2018, Chicago, IL, USA

(C) 2018 Copyright is held by the owner/author(s). Publication rights licensed to ACM.

ACM 978-1-4503-4953-6/18/03...\$15.00

https://doi.org/10.1145/3171221.3171281 robots yearly to work alongside employees across its 20 fulfillment centers $[46,56]$. In fact, robots are expected to replace as much as half the workforce in 10-20 years $[1,48,66]$.

Organizations like Amazon are now facing a new challenge of integrating humans and robots into one cohesive workforce. The challenge is made harder, in part, by the fear many workers have about their future employment [60]. There is a growing concern that robots are taking jobs away from humans [43, 60]. This fear has spread from blue-collar workers performing physical labor to white-collar workers performing more mentally intensive labor [36, 59]. This fear has engendered negative attitudes toward working with robots [43]. Many organizations are now seeking ways to help their employees overcome their negative attitudes toward working with robots [39]. Considering the importance of the topic, more theoretical and empirical work is needed [71].

Similarity between humans and robots is an important facilitator of positive attitudes toward robots $[35,68]$. The more an individual believes that a robot is similar to them, the more they like and prefer to interact with them $[5,54,61,70]$. For instance, research has found that individuals tend to like more and build stronger emotional attachment toward robots that appear to have a similar personality to theirs $[35,68]$. Therefore, the literature on human-robot similarity provides an excellent foundation to identifying the work conditions that may encourage humans to work with robots.

Although the research on human-robot similarity can be used to understand when people are likely to be willing to work with robots, several important areas still need to be explored. First, the prior research on human-robot similarity has paid little attention to whether or how the type of similarity may matter. For example, do we expect all types of similarity to have the same effect on one's willingness to work with robots? Second, we know very little about when such similarities are less important. More specifically, robots are often employed to perform very dangerous and in some cases undesirable tasks [2, $21,60]$. These tasks often have the potential to be physically harmful to humans. It is reasonable to assume that the degree of risk to physical danger might influence the importance of human-robot similarity. Yet, research exploring the impact of human-robot similarity has not incorporated the impacts of such risks. 
To address these shortcomings, we propose a research model (see Figure 1 on page 3). This research model identifies trust in a robot as a key mediating variable between human-robot similarity and one's willingness to work with a robot. It also differentiates between the surface-level human-robot similarity and the deep-level human-robot similarity. Surface-level similarity refers to similarity based on demographic characteristics that are typically more visible [24]. These represent social categories such as a robot's gender. Deep-level similarity represents similarity that is often less visible [24]. These include similarity in values, attitudes, beliefs and/or knowledge such as one's personality. The impact of the risk of physical danger is incorporated into the model as a boundary condition regarding both the impact of human-robot similarity on trust in a robot and the impact of one's willingness to work with a robot on one's willingness to work with a robot over a human co-worker.

To empirically examine this research model, we conducted an experimental study with 200 participants on Amazon Mechanical Turk. Participants were randomly assigned to one of the eight conditions using a 2 (surface-level similarity: same gender vs. different gender) x 2 (deep-level similarity: same work style vs. different work style) x 2 (risk of physical danger: high vs. low) between-subjects design. Participants were presented with a scenario where they were asked to imagine performing a collaborative task with an intelligent robot. Then they were asked a series of questions regarding their attitudes toward working with the robot under a specific set of conditions.

We had several interesting findings. First, the surface-level human-robot similarity in gender enhanced trust in a robotic coworker only when the risk of physical danger was low. However, the deep-level similarity in workstyle enhanced trust in the robotic co-worker regardless of the level of risk. Trust in the robot increased the participant's willingness to work with the robot. Willingness to work with the robot led to subsequent willingness to work with a robot over a human co-worker. However, this was somewhat moderated by the risk of physical danger. Participants were more willing to replace their human co-worker when the risks were high.

Based on the findings, this study contributes to human-robot interaction (HRI) research in the following ways. First, we highlight the importance of the type of similarity relative to the level of risk of physical danger. Second, we demonstrate that trust in the robot is vital to understanding the relationship between human-robot similarity and one's willingness to work with the robot. Third, we highlight the impact of the risk of physical danger as a moderator in understanding when similarity is likely to promote trust in the robot. Finally, we introduced the outcome variable willingness to work with a robot over a human co-worker. This dependent variable better reflects the desired outcome needed to help organizations overcome the challenge of integrating humans and robots into one cohesive workforce. Overall, this study has potential to inform the practice of human-robot collaboration and design of its robots.

\section{BACKGROUND}

\subsection{Similarity in HRI}

Similarity between co-workers has been a strong predictor of interpersonal work relationships [57]. In general, individuals prefer to interact and trust others they believe are similar to them [57, 63]. Similarity is often grouped into two categories: surface-level and deep-level [24, 47]. Surface-level similarity refers to similarities in visible physical characteristics such as gender, age, and race [24]. On the other hand, deep-level similarity is related to less visible characteristics such as knowledge, skills, values, and attitudes [24]. Surface-level and deep-level similarity have both been shown to promote positive perceptions between individuals and better work outcomes [12, 37].

Similarity has also shown to be central to understanding interaction with robots $[13,14]$. This research can also be categorized into surface-level and deep-level similarity. Surfacelevel similarity has led to positive attitudes toward robots [33]. Eyssel and Kuchenbrandt [14] found that people rated robots manufactured in their own country more positively and more humanlike. Another study, by Eyssel and Loughnan [16], showed that similarity could be invoked by a robot's projected gender and ethnicity.

HRI scholars have also examined what can be classified as deep-level similarity [41]. Research shows that people can form beliefs about a robot's personality or attitude based on simple conversational cues and behaviors [35, 68]. Bernier and Scassellati [5] demonstrated that robots that appear to have the same preference as their operators were rated as friendlier. Similarity with regard to personality is an important topic in HRI. Many studies have shown that when people believe that their robots are similar to them in personality, they are more motivated to interact with their robot, more engaged with the interaction with their robot, and do so for longer periods of time $[3,35,61,67]$.

\subsection{Trust in HRI}

Trust in robots has been an important topic in HRI [10, 19, 22]. The prior literature can be divided into factors that are related to the human and those related to the robots (see [22] for a review). Human-related factors include disposition to trust, negative attitudes toward robots, and individuals' personality [22, 29, 42, 44]. For instance, people with high levels of disposition to trust tend to trust robots more, whereas people with general negative attitudes toward robots tend to trust robots less [22, 44]. Kuchenbrandt and colleagues [34] found gender differences. Specifically, females tended to trust robots more than males. Haring et al. [23] found that people who were extroverts trusted robots more than introverts.

Robot-related factors that promote trust have also been found to be important. Particularly, a robot's performance influences whether humans trust it $[10,22,54]$. Trust was found to be a function of a robot's performance and its fluency in motions [6]. For instance, Desai et al. [11] reported that drops in reliability of robot autonomy decreased trust in a robot. It has also been found 
that robots that are functionality sophisticated are more trusted than robots with social abilities [19].

The literature on human-robot similarity and trust has provided new insights, but several areas call for further attention. First, little attention has been directed at examining whether similarity and trust can be leveraged to encourage someone to work with a robot, or at understanding when they might prefer to work with a robot over a human co-worker. Yet, both may be fundamental to identifying the conditions that encourage human-robot work collaboration. Second, no study has examined both surface-level and the deep-level similarities at the same time. Yet, one might be more or less important in the presence of the other [47]. Therefore, examining only one aspect of similarity can limit our understanding of how human-robot similarity influences our perceptions of robots. Third, we know little about the boundary conditions of the impacts of humanrobot similarity. Previous HRI findings suggest that future research begin to examine moderators [34, 40, 62]. Robots are deployed to work with humans on tasks that often involve physical danger [9]. From a human worker's perspective, risks related to physical danger are often viewed as a more important motivator for working with robots than other types of risk (e.g., financial cost) [2]. This would suggest the need to investigate the risk of physical danger as a potential moderator in human-robot collaboration. This area should become more important because robots are expected to be involved in $30-45 \%$ of all work in the United States by 2025 [58]. Nonetheless, it is unclear whether individuals' positive attitudes toward working with robots lead to the preference for a robotic co-worker over a human.

\section{RESEARCH MODEL AND HYPOTHESES}

We put forth a research model in which the surface-level and the deep-level similarity increase trust in the robot, intention to work with the robot, and intention to work with a robot over a human co-worker. These relationships are moderated by the risk of physical danger. A summary of these arguments is presented in Figure 1.

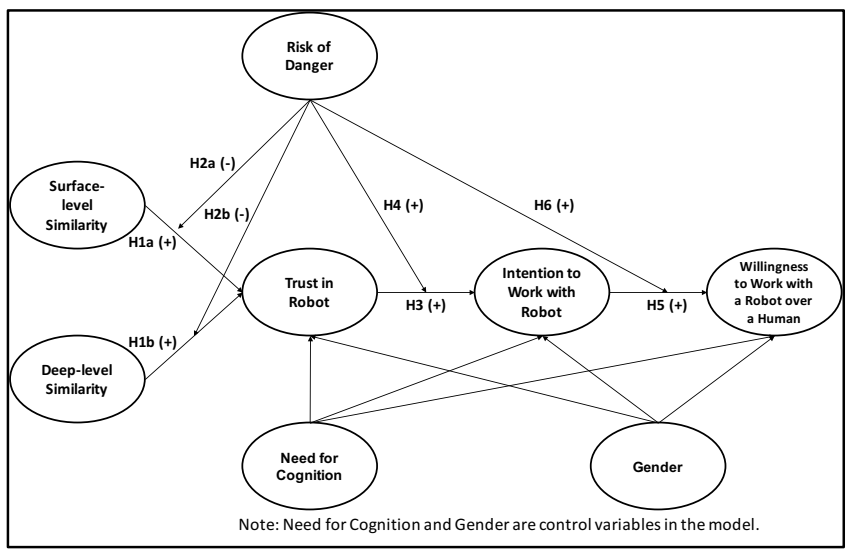

Figure 1: Research Model
The first hypothesis proposes that higher levels of similarity between a human and a robot will foster trust in the robot. Trust in robots has been viewed to include both interpersonal and technological trust due to robots' physical embodiment, which manifests human attributes [21, 22]. Thus, this study employs a conceptualization of trust in a robot that involves both interpersonal and technological aspects of trust: an individual's willingness to be vulnerable to and dependent on the robot.

The idea that similarity can increase trust in a robot is based on the similarity and attraction theory (SAT) [7, 35, 57]. SAT asserts that individuals are more attracted to those whom they believe are similar to them [7, 57]. They often base their judgment of similarity on gender and personality [50, 57]. Individuals who are believed to be similar are often considered to be more trustworthy, while those that are not similar are considered less trustworthy $[50,55]$.

Similarity can be based on both surface-level and deep-level characteristics. For surface-level similarity among humans, gender is one of the most salient surface-level cues [53]. Gender has been found to be an important cue for similarity between humans and robots. For instance, Eyssel et al. [15] reported that gender similarity between a robot and an individual led to more positive feelings and psychological closeness. Deep-level similarity between humans and robots has also been shown to have positive impacts [5]. Andrist et al. [3] found that matching a user's and a robot's personality led to more positive evaluation of the robot. Similarity in personality has also been shown to promote trust in robots [5,67]. Therefore, the surface-level and the deep-level similarity should promote trust in a robot coworker.

H1) (a) Surface-level similarity and (b) deep-level similarity are positively related to trust in the robot.

The risk of physical danger should moderate the positive effects of similarity on trust in the robot. The risk of physical danger is a situational moderator, which is related to the nature of work performed by a human and a robot $[52,60]$. When the risk of physical danger is low, individuals are likely to build trust based on perceptions of similarity. In these situations, individuals have less need to assess anything other than the salient similarity cues, so the similarity should have a strong impact on individuals' trust in their robot co-worker. This might explain why robotic pets and rehabilitation robots, used in lowrisk situations, are preferred when they demonstrate similarities in things like appearance rather sophisticated technical features and computational power $[18,35,68]$.

On the other hand, when the risk is high, the positive link between similarity and trust in the robot weakens. When the risk of physical danger is high, individuals rely less on the similarity to determine trust in the robot. Individuals rely on the robot's capabilities rather than just how similar it is when assessing their level of trust in the robot. For instance, aspects other than similarities, such as the robot's technical specifications and intelligence, also come into play in determining trust in the robot. This means that individuals rely less on similarity to determine trust in their robotic co-worker. In this respect, Groom and Nass [21] argued that trust in a robotic teammate should not simply be a function of liking of the robot and that various factors should be considered to ensure safety and trust in high-stakes situations like space missions and military operations. As such, we hypothesize that: 
H2) The risk of physical danger moderates the effects of (a) surface-level similarity and (b) deep-level similarity on trust in a robot, such that increases in risk weaken the relationship between both types of similarity and trust.

The heightened trust in the robot leads to greater intention to work with the robot as a team. This is in part because trust in the robot creates positive attitudes toward the robot $[19,62]$. Positive attitudes toward the robot include reduced fear of failure of the robot's functionality and reduced concern that working with the robot will require consistent and effortful monitoring [19, 42, 65]. Moreover, trust in the robot reduces uncertainty about the robot's behavior and helps enhance a feeling of control over interactions with the robot [19]. The sense of control is also an element of positive attitudes that result in greater behavioral intention [51]. In sum, trust in a robot promotes positive attitudes and a sense of control by reducing uncertainty and generating expectations of positive experiences, which result in intention to work with the robot.

H3) Trust in the robot is positively related to intention to work cooperatively with the robot.

However, the positive link between trust in a robot and intention to work with the robot may not be uniform in all circumstances. The risk of physical danger regulates the impact of trust in the robot on one's intention to work with the robot. Specifically, when the risk of danger is present, trust in a robot demonstrates a stronger impact on intention to work with the robot. As stated, the risk of danger alters an individual's cognitive process and dictates what cognitive resources influence intention to work with a robot $[21,38]$. When there is a higher risk of danger, individuals perceive greater uncertainty in the task and seek ways to regain the perception of control [20, 45]. In this case, the role of trust in a robot becomes more salient as a cognitive basis for reducing uncertainty and maintaining control. Therefore, the effect of trust in a robot on intention to work with the robot is stronger in high-risk situations.

H4) The risk of physical danger moderates the relationship between trust in a robot and intention to work with the robot, such that the effect is stronger when risk is higher.

Using the research model, we examined whether the intention to work with the robot promotes the willingness to work with a robot over a human co-worker. It seems natural to speculate that the greater the intention to work with the robot is, the more likely it is that an individual will reveal a stronger preference for robots. A strong preference for robots is an indicator that an individual might choose a robot over a human teammate.

H5) Intention to work with a robot is positively related to willingness to work with a robot over a human.

Risk of physical danger should moderate the impact of intention to work with a robot on willingness to work with a robot over a human co-worker. The risk of danger triggers the deliberate and conscious cognitive process when judging whether to work with a robot or with a human teammate [38, 50]. In this case, individuals might conclude that it is better to deploy robots to a risky and dangerous situation than to risk precious human lives. Based on this judgment, individuals will perceive that working with the robot is more beneficial than risking human safety. On the other hand, when the risk is low, the positive impact of intention to work with the robot on the willingness to work with a robot over a human co-worker might be weaker, or not present. Low-risk situations are less likely to trigger and will not make individuals engage in a careful reasoning when choosing between working with a human and working with a robot $[38,50]$. When the risk is low, there might be no potential benefit of working with a robot because no teammates have to risk their lives.

H6) The risk of danger moderates the relationship between intention to work with a robot and willingness to work with a robot over a human co-worker, such that the relationship is stronger when the risk is high than when the risk is low.

\section{METHOD}

We conducted a 2 (surface-level similarity: the same gender vs. different gender) x 2 (deep-level similarity: same work style vs. different work style) x 2 (risk of danger: high vs. low) betweensubjects online experiment. In the experiment, participants were randomly assigned to one of the eight conditions and viewed a video about a robot and a hypothetical scenario, in which collaboration between a human and a robot would be essential.

\subsection{Participants}

We recruited a total of 200 participants $(77$ male; mean age $=$ 36.5 years, standard deviation $[\mathrm{SD}]=10.77$ years; min. age $=18$ years, max. age $=68$ years) through Amazon Mechanical Turk (MTurk). Individual participants completed a short self-report questionnaire individually and were paid at the completion of an experimental session. The sample consisted of people of diverse education levels, ages, genders, and ethnicities. The sample included MTurk workers in the United States with good performance histories (having 95\% or more of their previous online tasks marked as high quality by requesters). The sample turns out to have been ethnically diverse: $64 \%$ White, $10 \%$ Asian, 8\% Black and African American, 6\% Hispanic and Latino, with the rest including Native American or Alaskan Native and Native Hawaiian.

\subsection{Scenario and Robot}

This study employed a hypothetical scenario where participants would be working with a robot collaboratively in a warehouse. A PR2 robot was used for the videos (Figure 2). The robot was chosen based on several criteria. First, the robot was genderneutral in its appearance. This is because the robot's gender was going to be manipulated only through its voice and name, ruling out any visual aspects of robots that might influence individuals' gender perception. Second, the form of the robot should imply some degree of motor abilities such as navigating and moving objects from one place to another location. The hypothetical scenario in the online experiment involved physical tasks, so it was important to use robots that could complete such tasks to provide believable portrayals of a robot and an individual working together. The identical robot was used for all participants across the different experimental conditions. 


\subsection{Experimental Manipulations}

Surface-level similarity had two conditions: same gender vs. different gender between an individual and the robot. Robot gender was manipulated using video clips that contained a synthesized computer voice and a name suggesting a typical gender attribution. Specifically, the female robot had a female voice and had the model name "RX-01 Jessica," whereas the male robot had a male voice and was named "RX-01 David." The videos were not skippable and were 40 seconds long.

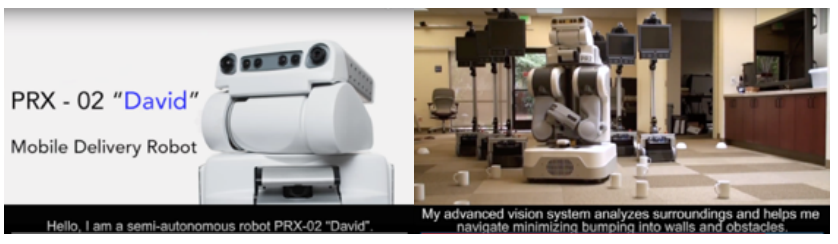

Figure 2: Screenshots from videos for the surface-level similarity manipulation.

Deep-level similarity had two levels: the same work style vs. a different work style. Individual participants were given a series of nine questions regarding work-style preferences generated based on work-style dimensions in Zellmer-Bruhn et al. [73]. In the same work-style condition, a robot chose the same answer as the participant after the participant made his or her choice, and showed the sentence, "I also chose the same statement. Your answer was [It is not okay to be 20 minutes late for a meeting because others team members' time will be wasted due to the delay]. My answer was [It is not okay to be 20 minutes late for a meeting because others team members' time will be wasted due to the delay]." On the other hand, in the different work-style condition, a robot chose the other answer and showed the opposite choice to the participant.

The risk of danger in the task was manipulated to have two levels: high risk and low risk. In the high-risk condition, participants were given a scenario with images, in which they had to collaborate with the robot to clear an area by loading highly toxic and hazardous containers onto a truck for disposal. In the low-risk condition, participants were given a similar scenario with images, but with wooden boxes to load onto the truck for home delivery.

\subsection{Procedure}

Participants were greeted and asked to fill out a consent form. To ensure that participants' paid attention during the experiment, we used several questions that asked specific details about the study's manipulations. On the first page of the experiment, participants were made aware that they would be asked detailed questions about the experiment to ensure that they paid attention. Then, they were given brief instructions about the experimental procedure and task. Next, they completed a pre-task questionnaire that included questions regarding demographic information including gender, age, and ethnicity, and control variables.
Then, participants were randomly assigned to either the same-gender condition or different-gender condition based on the gender information indicated in the pre-task questionnaire. Next, they were asked to choose responses to the nine questions about work styles. Immediately after the participant chose a response to a question, the robot's choice was shown on the following screen next to the participant's choice, according to the condition they were assigned. Once all the questions were shown, a summary table that compared the robot's and the participant's answers to all questions was given to the participants. Participants were then asked to view a scenario about collaboration with the robot with accompanying illustrative images. It was expected to take 5-10 minutes for participants to view the scenarios.

Finally, participants were asked to fill out a post-task questionnaire, which included dependent measures such as trust in the robot, intention to work with a robot cooperatively, and willingness to work with a robot over a human co-worker.

\subsection{Measures}

4.5.1 Manipulation Check Measures. To check the success of the surface-level similarity, participants were asked which gender they thought the robot was after the video was shown. All participants answered the robot's gender correctly according to the gender in the video, which indicates the successful manipulation. Deep-level similarity was ensured by a series of five of questions regarding perceived similarity in work style adopted from [73] based on a 5-point scale. Example items include "The robot has similar work habits with me," and "The robot has similar interaction styles with me." The scale was reliable (Cronbach's $\alpha=0.98$ ). Results of a $t$-test showed that perceived similarity in work style was significantly higher in the same-work-style condition $(\mathrm{M}=4.29, \mathrm{SD}=0.83)$ than in the different-work-style condition $(\mathrm{M}=1.69, \mathrm{SD}=0.77)(\mathrm{t}(198)=$ 22.99, $\mathrm{p}<0.001)$.

As a manipulation check for the risk of physical danger, perceived risk of physical danger was measured using an index of four items adapted from [31] and [27] based on a 5-point scale. Examples include "I will encounter personally hazardous situations during the task when I work with the robot" and "The task seems to be risky." The scale was reliable $(\alpha=0.91)$. Perceived risk of danger was significantly higher in the high-risk condition $(\mathrm{M}=4.58, \mathrm{SD}=0.50)$ than in the low-risk condition $(\mathrm{M}$ $=3.42, \mathrm{SD}=0.76)(\mathrm{t}(198)=12.71, \mathrm{p}<0.001)$.

4.5.2 Control Variables. We collected age, gender, and ethnicity of participants and Negative Attitudes toward Robots Scale (NARS) ratings [43]. Also, we measured the dispositional need for cognition using an index of 14 items adapted from Cacioppo et al. [8] based on a 5-point scale. The scale captures the degree to which an individual participant is likely to engage in cognitive processes in general [8,30]. Sample items include "I really enjoy a task that involves coming up with new solutions to problem." The scale was reliable (Cronbach's $\alpha=0.96$ ).

4.5.3 Dependent Measures. We measured trust in the robot to capture the degree to which an individual believed the robot was dependable and trustworthy. The scale consisted of eight items 
adapted from Jian et al. [28] and was measured using a 5-point scale. The questions included items such as "I am able to trust the robot," and "The robot is reliable." The scale was reliable (Cronbach's $\alpha=0.92$ ).

We measured intention to work with the robot to capture an individual's willingness to admit the robot as a team member and work together as a team. An index of five items was adapted from Venkatesh and Davis [64] based on a 5-point scale. The questions included "Assuming I had another project similar to this one and access to this robot, I am willing to work with this robot as a team," and "This robot and I will likely make a good team." The scale was reliable (Cronbach's $\alpha=0.95$ ).

Finally, we measured willingness to work with a robot over a human co-worker to capture the degree to which an individual wanted to work with the robot instead of a human teammate. We developed an index of three items that were measured using a 5-point scale. The three items included "For this job, I would prefer to work with the robot instead of a human," "For this job, I would rather replace a human with the robot," and "For this job, I would rather team up with the robot than a human." The scale was reliable (Cronbach's $\alpha=0.83$ ).

\section{RESULTS}

All analyses in the following section were conducted by following the partial least squares (PLS) approach using SmartPLS 3.2. Age, ethnicity, and NARS were not significant and were excluded from results.

\subsection{Measurement Validity}

We assessed discriminant validity of the measures based on a factor analysis. All items loaded at 0.70 or above on each of their constructs and indicated no cross-loadings above 0.4 . The results of the factor analysis indicate discriminant and convergent validity of the measurable latent variables in the model [17]. The correlation matrix, shown in Table 1, indicates that correlations among all constructs were well below the square roots of the average variance extracted (AVE). Finally, the internal consistency of the variables was assessed by calculating internal composite reliability (ICR). All variables indicated values well above 0.70 , which is evidence of internal consistency.

Table 1 Descriptive statistics and correlations

\begin{tabular}{|c|c|c|c|c|c|c|c|c|c|c|}
\hline Variable & Mean & SD & 1 & 2 & 3 & 4 & 5 & 6 & 7 & 8 \\
\hline 1. Gender & 0.39 & 0.49 & NA & & & & & & & \\
\hline 2. Need for Cognition (NCOG) & 3.50 & 0.91 & $-0.21 * *$ & $\begin{array}{c}0.80 \\
(0.96) \\
\end{array}$ & & & & & & \\
\hline 3. Surface-level Similarity (SLS) & 0.45 & 0.50 & -0.26 & 0.06 & NA & & & & & \\
\hline 4. Deep-level Similarity (DLS) & 0.51 & 0.50 & 0.23 & 0.04 & -0.4 & $\mathrm{NA}$ & & & & \\
\hline 5. Risk of Physical Danger (RPD) & 0.49 & 0.50 & 0.67 & 0.05 & 0.03 & 0.07 & NA & & & \\
\hline 6. Trust in Robot (TR) & 3.71 & 0.76 & 0.11 & 0.12 & -0.03 & $0.38 * *$ & $-0.15^{*}$ & \begin{tabular}{|c|}
0.79 \\
$(0.94)$
\end{tabular} & & \\
\hline $\begin{array}{l}\text { 7. Intention to Work with the } \\
\text { Robot (IWR) }\end{array}$ & 4.23 & 0.80 & 0.11 & $0.16^{*}$ & -0.01 & $0.24 * *$ & -0.03 & $0.56 * *$ & $\begin{array}{c}0.92 \\
(0.96)\end{array}$ & \\
\hline \begin{tabular}{|l|} 
8. Intention to Replace a Human \\
with the Robot (IRHR)
\end{tabular} & 3.50 & 1.00 & $0.14 *$ & 0.06 & 0.05 & $0.19 * *$ & $0.14 *$ & $0.33^{* *}$ & $0.52^{* * *}$ & $\begin{array}{c}0.86 \\
(0.90) \\
\end{array}$ \\
\hline \multicolumn{11}{|c|}{ 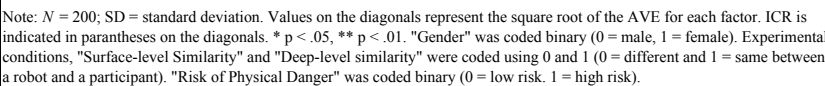 } \\
\hline
\end{tabular}

6

\subsection{Hypothesis Testing}

H1 posited that (a) surface-level and (b) deep-level similarity would increase trust in the robot, respectively. Surface-level similarity did not increase trust in the $\operatorname{robot}(\beta=-0.01, \mathrm{p}=0.87)$. However, there was a significant positive impact of deep-level similarity on trust in the robot $(\beta=0.39, \mathrm{p}<0.001)$, which indicates that only $\mathrm{H} 1 \mathrm{~b}$ was supported.

$\mathrm{H} 2 \mathrm{a}$ and $\mathrm{H} 2 \mathrm{~b}$ posited moderation effects of the risk of danger for the relationships between surface-level and deep-level similarity and trust in a robot, respectively. There was a significant interaction effect between surface-level similarity and the risk of danger in predicting trust in the robot $(\beta=-0.17, \mathrm{p}<$ 0.01 ). As hypothesized in H2a, the risk of danger moderated the impact of surface-level similarity on trust in the robot, such that the positive impact of surface-level similarity was found only in the low-risk condition (Figure 3). However, an interaction effect was not found between deep-level similarity and trust in the robot $(B=0.05, \mathrm{p}=0.48)$. Thus, only H2a was supported.

H3 hypothesized that trust in the robot would increase an individual's intention to work with the robot as a team. H3 was fully supported based on the significant path coefficient $(B=$ $0.58, \mathrm{p}<0.001)$. H4 posited a moderation effect of risk of danger for the relationship between trust in the robot and intention to work with the robot, such that the positive impact of trust in the robot would be stronger in the high-risk condition. H4 was not supported $(B=-0.08, p=0.22)$. H5 posited the positive impact of intention to work with the robot on the individual's willingness to work with a robot over a human co-worker. H5 was fully supported $(B=0.55, \mathrm{p}<0.001)$.

Finally, H6 posited that the positive impact of an intention to work with the robot would be strengthened in the high-risk condition, whereas the impact would not be present or would be weakened in the low-risk condition. The model demonstrated a marginally significant interaction effect $(\beta=0.09, \mathrm{p}<0.1)$. The interaction effect was tested by a separate analysis employing a linear regression. A plot based on the results of the regression analysis showed that intention to work with the robot increased the willingness to work with a robot over a human co-worker only in the high-risk condition $(\beta=0.32, \mathrm{p}<0.05)$ (Figure 4 ). Thus, H6 was partially supported.

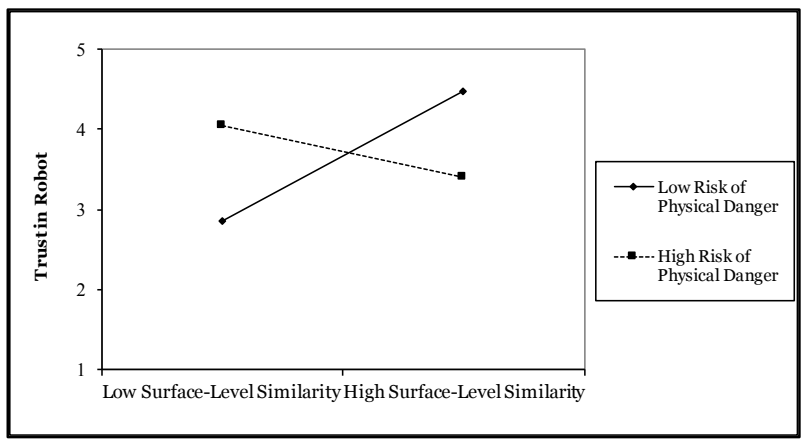

Figure 3: Moderation effect of risk of danger for the relationship between the surface-level similarity and trust in the robot 


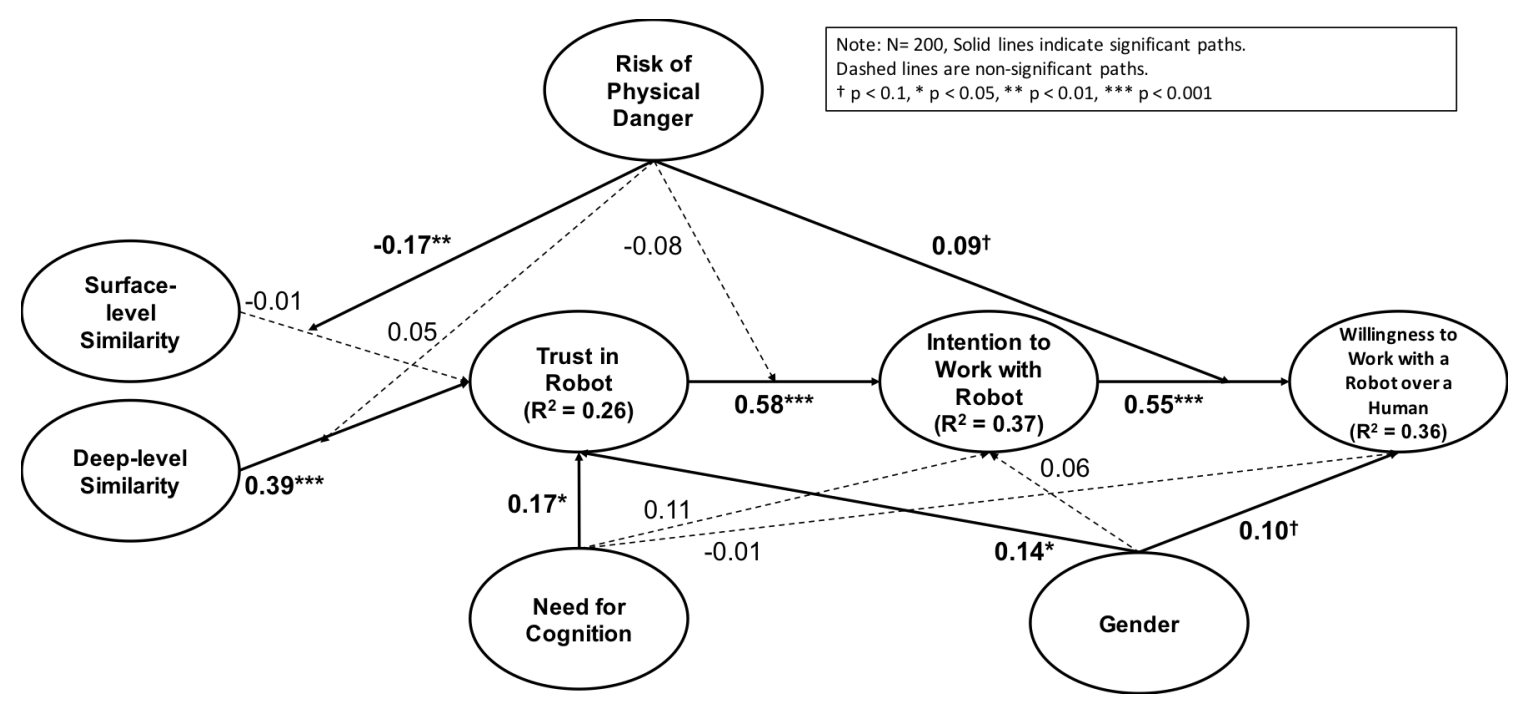

Figure 5: Results of PLS analysis

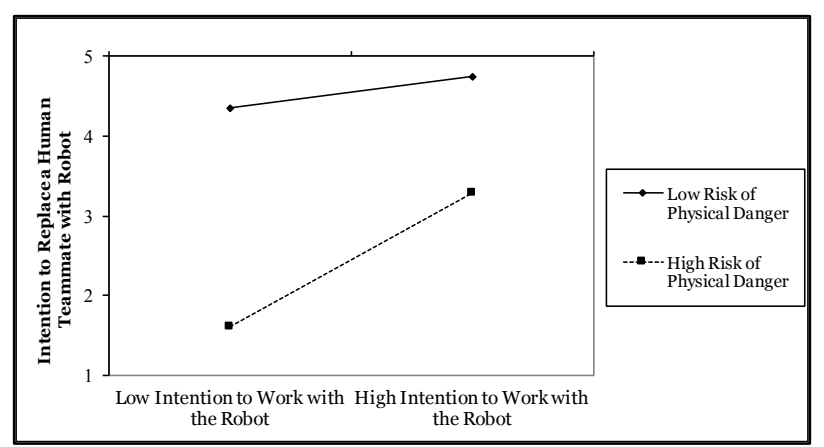

Figure 4: Moderation effect of risk of danger for the relationship between intention to work with the robot and willingness to work with a robot over a human co-worker

Based on the hypothesis testing, the final model was derived from the research model (Figure 5). The model illustrates the results of the model testing, where $\mathrm{R}^{2}$ indicates the variance explained and $\beta$ indicates the standardized path coefficients of each path in the structural model. $\mathrm{R}^{2}$ indicates that trust in the robot was explained by $26 \%$. Intention to work with the robot and willingness to work with a robot over a human co-worker were explained by $37 \%$ and $36 \%$, respectively.

\section{DISCUSSION}

Our goal was to identify ways to help employees overcome their negative attitudes toward working with robots. Results from an online experiment showed that surface-level similarity increased trust in a robot, but only when the risk was low. However, deeplevel similarity increased trust in the robot regardless of the risk. Trust in the robot was found to increase intention to work with the robot and subsequently willingness to work with a robot over a human co-worker. The risk of physical danger also marginally moderated the relationship between intention to work with the robot and willingness to work with a robot over a human co-worker. This study has several implications for research and practice.

\subsection{Implications for Research}

First, our findings contribute to the literature on perceptions of robot similarity. This literature has not focused on whether the type of similarity matters with regard to human-robot interaction (e.g., [3, 5, 33]). We demonstrate that the type of similarities should be taken into account when trying to encourage individuals to work with a robot. Under the conditions of low physical risk, both surface-level and deep-level similarity can be used to promote trust in a robot. However, when the risk of physical danger was high, surface-level similarity had no impact on trust. Our findings showed that risk of physical danger has a role in moderating the relationships among human-robot similarity and trust in a robot. Identifying the boundary condition related to the surface-level similarity is especially vital for work settings. Unlike social robots, which are mostly deployed to safe environments like homes, robots used in work settings may be required to fulfill dangerous tasks with a higher risk of physical danger. In doing so, we highlight the importance of deep-level similarity over surface-level similarity in conditions of high risk.

This finding calls for future research in several important areas. Future research seeking to promote trust in robots through similarity should consider the risk of physical danger. More specifically, future research should focus on the deep-level similarity when robotic co-workers are involved in dangerous tasks. Future research should identify other factors that alter the impacts of similarity, such as task interdependence, task duration, and competitive structure of the task. For instance, do similarity effects on trust in a robot change over time after a few initial interactions with the robot? Also, similarity with a robot 
may yield a negative perception of the robot when someone is competing with the robot rather than cooperating [40].

Second, our findings contribute to the literature on robot acceptance. Prior researchers have paid little attention to the potential moderating role of risk in human-robot interaction (e.g., $[19,26])$. Yet, robots are often employed to perform very dangerous tasks $[2,60]$. Therefore, it is reasonable to assume that the degree of risk to physical danger could be important. Despite this, results of this study highlight that risk did not moderate the relationship between trust and intention to work with the robot. This was contrary to our speculation that risk would amplify the importance of trust. This implies that trust is an essential and salient element to promoting workers' attitudes toward robotic co-workers regardless of the risk involved in the interaction. Trust appears to be a much more robust facilitator of intention to work with a robot than we originally thought. Future research should be done to determine whether the type of trust matters. For example, different types of trust (i.e., affect vs. cognitive) might be more or less robust in the presence of risk.

Finally, our results extend the literature on intention to work with or interact with a robot. Unlike prior literature (see [15]), we examined the moderation effect of risk on the relationship between intention to work with and the willingness to work with a robot over a human co-worker. Our results showed that the more individuals are willing to work with the robot, the more likely they are to choose a robot over a human co-worker. However, the results also demonstrated that this phenomenon is somewhat governed by risk. Intention to work with a robot had a much stronger relationship with willingness to work with a robot over a human co-worker when the task was considered to be high in physical risk. These results suggest that humans do account for the level of risk when determining whether a human co-worker should be replaced. We also highlight that the willingness to work with a robot over a human co-worker is conceptually distinct from an intention to work with a robot. Unlike an intention to work with a robot that is determined solely by a robot's characteristics, the willingness to work with a robot over a human co-worker may address the comparative benefit of working with a robot. Future research should identify other important moderators. This might include the guilt of undermining employment opportunities for other human coworkers.

\subsection{Implications for Practice}

First, robots deployed to work with humans should be designed to display similarity with the humans to ensure higher levels of trust and intention to work with the robots. Given that deeplevel similarity yielded a strong impact, robots should be designed to manifest similarity with workers on a deep level, such as work styles, values, preferences, and personality. For instance, designers could embed a conversational protocol to a robot's software and customize it to express similar preferences on things that a worker liked. Designers could also employ surface-level similarity to robots. For instance, similar color schemes and logos are effective visual cues to highlight surfacelevel similarity. However, the application of surface-level similarity should take into account that it can work only in lowrisk situations.

Second, managers of teams working with robots should be wary of the level of risk in a workplace where a robot and an individual collaborate with each other. In many cases, decisions about adopting a robot in a work environment are made at an executive level and may not reflect individual workers' intention to work with them. Based on our results, teams should be knowledgeable about the level of risk in the task where a robot is deployed. Particularly when robots replace human laborers and become part of human-robot teams, managers of such teams should be aware that merely highlighting some similarity with their employee will not necessarily result in greater intention to choose to work with a robot instead of a human teammate.

\subsection{Limitations}

There are several limitations in this study. First, this study was conducted through an online experiment that involved interacting with a robot by watching a pre-recorded video. Results might differ in magnitude if an individual interacted directly with a robot viewing a scenario with potential interactions. Second, we should also note that our participants were MTurkers. There has been much discussion with regard to the use of MTurkers. Many studies have found MTurkers to be a valid and appropriate sample [4, 25]. Nonetheless, like all studies, we caution against over-generalizing our findings to broader contexts. Third, the context of this study involved only one robot and one person per collaborative working relationship. Yet, such collaborative relationships with robots can include more than one person and more than one robot $[70,71]$. These relationships can also be much more dynamic in many cases [69, 71]. Finally, this study examined only one aspect of surface-level and deeplevel diversity, respectively. Perceptions of similarity can be elicited by many factors other than gender and work style, such as place of origin, ad-hoc membership, abilities, and knowledge $[33,49]$.

\section{CONCLUSION}

It is vital for teams working with robots to attain positive attitudes regarding the robots and working with them in order to succeed. This study examined the impacts of similarity between an individual and a robot on fostering trust in the robot and intention to work with the robot in tasks of different levels of danger risk. Results showed that the positive impacts of similarity are contingent upon the degree of danger risk in a task. Results also demonstrated that trust in a robot positively predicts subsequent intention to work with the robot and willingness to work with a robot over a human co-worker.

\section{REFERENCES}

[1] Evan Ackerman. 2014. U.S. Army Considers Replacing Thousands of Soldiers with Robots. (Jan. 22, 2014). Retrieved Jan. 3, 2018 from https://spectrum.ieee.org/automaton/robotics/military-robots/army-considersreplacing-thousands-of-soldiers-with-robots

[2] Obehioye Adubor, Rhomni St. John, and Aaron Steinfeld. 2017. Personal Safety is More Important Than Cost of Damage During Robot Failure. In Proceedings 
of the Companion of the 2017 ACM/IEEE International Conference on HumanRobot Interaction, 403-403.

[3] Sean Andrist, Bilge Mutlu, and Adriana Tapus. 2015. Look Like Me: Matching Robot Personality via Gaze to Increase Motivation. In Proceedings of the 33rd Annual ACM Conference on Human Factors in Computing Systems, 3603-3612.

[4] Adam J. Berinsky, Gregory A. Huber, and Gabriel S. Lenz. 2012. Evaluating online labor markets for experimental research: Amazon.com's Mechanical Turk. Polit. Anal. 20, 3 (2012), 351-368.

[5] Emily P. Bernier and Brian Scassellati. 2010. The Similarity-attraction Effect in Human-Robot Interaction. In Development and Learning (ICDL), 2010 IEEE 9th International Conference on, 286-290.

[6] Rik van den Brule, Ron Dotsch, Gijsbert Bijlstra, Daniel H. J. Wigboldus, and Pim Haselager. 2014. Do robot performance and behavioral style affect human trust? Int. F. Soc. Robot. 6, 4 (November 2014), 519-531.

[7] D. E. Byrne. 1971. The Attraction Paradigm, Vol. 11. Academic Press, Cambridge, MA.

[8] John T. Cacioppo, Richard E. Petty, Jeffrey A. Feinstein, and W. Blair G. Jarvis. 1996. Dispositional differences in cognitive motivation: The life and times of individuals varying in need for cognition. Psychol. Bull. 119, 2 (1996), 197.

[9] Agostino De Santis, Bruno Siciliano, Alessandro De Luca, and Antonio Bicchi 2008. An atlas of physical human-robot interaction. Mech. Mach. Theory 43, 3 (2008), 253-270.

[10] Munjal Desai, Poornima Kaniarasu, Mikhail Medvedev, Aaron Steinfeld, and Holly Yanco. 2013. Impact of Robot Failures and Feedback on Real-time Trust. In Proceedings of the 8th ACM/IEEE International Conference on Human-robot Interaction (HRI '13), 251-258.

[11] Munjal Desai, Mikhail Medvedev, Marynel Vázquez, Sean McSheehy, Sofia Gadea-Omelchenko, Christian Bruggeman, Aaron Steinfeld, and Holly Yanco. 2012. Effects of Changing Reliability on Trust of Robot Systems. In Proceedings of the Seventh Annual ACM/IEEE International Conference on Human-Robot Interaction (HRI '12), 73-80. DOI:https://doi.org/10.1145/2157689.2157702

[12] IJ Hetty van Emmerik and Veerle Brenninkmeijer. 2009. Deep-level similarity and group social capital: Associations with team functioning. Small Group Res. 40, 6 (2009), 650-669.

[13] Friederike Eyssel and Dieta Kuchenbrandt. 2011. Manipulating Anthropomorphic Inferences about NAO: The Role of Situational and Dispositional Aspects of Effectance Motivation. In RO-MAN, 2011 IEEE, 467472.

[14] Friederike Eyssel and Dieta Kuchenbrandt. 2012. Social categorization of social robots: Anthropomorphism as a function of robot group membership. Br. F. Soc Psychol. 51, 4 (2012), 724-731.

[15] Friederike Eyssel, Dieta Kuchenbrandt, Simon Bobinger, Laura de Ruiter, and Frank Hegel. 2012. "If You Sound Like Me, You Must Be More Human": On the Interplay of Robot and User Features on Human-robot Acceptance and Anthropomorphism. In Proceedings of the Seventh Annual ACM/IEEE International Conference on Human-Robot Interaction (HRI '12), 125-126. DOI:https://doi.org/10.1145/2157689.2157717

[16] Friederike Eyssel and Steve Loughnan. 2013. “It Don't Matter If You're Black or White"? In ICSR 2013: Social Robotics. Springer, New York, NY, 422-431.

[17] Claes Fornell and David F. Larcker. 1981. Structural equation models with unobservable variables and measurement error: Algebra and statistics. F. Mark Res. (1981), 382-388.

[18] Batya Friedman, Peter H. Kahn Jr., and Jennifer Hagman. 2003. Hardware Companions?: What Online AIBO Discussion Forums Reveal about the Human-Robotic Relationship. In Proceedings of the SIGCHI Conference on Human Factors in Computing Systems, 273-280.

[19] Ilaria Gaudiello, Elisabetta Zibetti, Sébastien Lefort, Mohamed Chetouani, and Serena Ivaldi. 2016. Trust as indicator of robot functional and social acceptance. An experimental study on user conformation to iCub answers. Comput. Hum. Behav. 61, (August 2016), 633-655. DOI:https://doi.org/10.1016/j.chb.2016.03.057

[20] David Gefen. 2000. E-commerce: the role of familiarity and trust. Omega 28, 6 (2000), 725-737.

[21] Victoria Groom and Clifford Nass. 2007. Can robots be teammates? Benchmarks in human-robot teams. Interact. Stud. 8, 3 (2007), 483-500.

[22] Peter A. Hancock, Deborah R. Billings, Kristin E. Schaefer, Jessie Y. C. Chen, Ewart J. De Visser, and Raja Parasuraman. 2011. A meta-analysis of factors affecting trust in human-robot interaction. Hum. Factors 7. Hum. Factors Ergon. Soc. 53, 5 (2011), 517-527.

[23] Kerstin Sophie Haring, Yoshio Matsumoto, and Katsumi Watanabe. 2013. How do People Perceive and Trust a Lifelike Robot? In Proceedings of the World Congress on Engineering and Computer Science.

[24] David A. Harrison, Kenneth H. Price, Joanne H. Gavin, and Anna T. Florey. 2002. Time, teams, and task performance: Changing effects of surface- and deep-level diversity on group functioning. Acad. Manage. 7. 45, 5 (2002), 10291045

[25] David J. Hauser and Norbert Schwarz. 2016. Attentive Turkers: MTurk participants perform better on online attention checks than do subject pool participants. Behav. Res. Methods 48, 1 (2016), 400-407.
[26] Marcel Heerink, Ben Kröse, Vanessa Evers, and Bob Wielinga. 2006. The Influence of a Robot's Social Abilities on Acceptance by Elderly Users. In Robot and Human Interactive Communication, 2006. ROMAN 2006. The 15th IEEE International Symposium on, 521-526.

[27] John M. Jermier, Jeannie Gaines, and Nancy J. McIntosh. 1989. Reactions to physically dangerous work: A conceptual and empirical analysis. 7. Organ. Behav. 10, 1 (1989), 15-33.

[28] Jiun-Yin Jian, Ann M. Bisantz, and Colin G. Drury. 2000. Foundations for an empirically determined scale of trust in automated systems. Int. 7. Cogn. Ergon. 4, 1 (2000), 53-71.

[29] M. P. Joosse, M. Lohse, J. Gallego Pérez, and Vanessa Evers. 2013. What You Do is Who You Are: The Role of Task Context in Perceived Social Robot Personality. In Robotics and Automation (ICRA), 2013 IEEE International Conference on. DOI:https://doi.org/10.1109/ICRA.2013.6630863

[30] Eric Kearney, Diether Gebert, and Sven C. Voelpel. 2009. When and how diversity benefits teams: The importance of team members' need for cognition. Acad. Manage. 7. 52, 3 (2009), 581-598.

[31] Sara Kim and Ann L. McGill. 2011. Gaming with Mr. Slot or gaming the slot machine? Power, anthropomorphism, and risk perception. f. Consum. Res. 38, 1 (June 2011), 94-107. DOI:https://doi.org/10.1086/658148

[32] Will Knight. 2015. Are You Ready for a Robot Colleague? (Sept. 28, 2015). Retrieved Jan. 3, 2018 from https://www.technologyreview.com/s/541676/areyou-ready-for-a-robot-colleague/

[33] Dieta Kuchenbrandt, Friederike Eyssel, Simon Bobinger, and Maria Neufeld. 2013. When a robot's group membership matters. Int. F. Soc. Robot. 5, 3 (2013), 409-417.

[34] Dieta Kuchenbrandt, Markus Häring, Jessica Eichberg, and Friederike Eyssel. 2012. Keep an Eye on the Task! How Gender Typicality of Tasks Influence [sic] Human-Robot Interactions. In International Conference on Social Robotics, Springer, 448-457.

[35] Kwan Min Lee, Wei Peng, Seung-A. Jin, and Chang Yan. 2006. Can robots manifest personality?: An empirical test of personality recognition, social responses, and social presence in human-robot interaction. f. Commun. 56, 4 (2006), 754-772.

[36] Clair Cain Miller. 2016. The long-term jobs killer is not China. It's automation. (Dec. 21, 2016). Retrieved Jan. 3, 2018 from https://www.nytimes.com/2016/12/21/upshot/the-long-term-jobs-killer-is-notchina-its-automation.html

[37] Susan Mohammed and Linda C. Angell. 2004. Surface-and deep-level diversity in workgroups: Examining the moderating effects of team orientation and team process on relationship conflict. F. Organ. Behav. 25, 8 (2004), 1015-1039.

[38] Kanchan Mukherjee. 2010. A dual system model of preferences under risk Psychol. Rev. 117, 1 (2010), 243.

[39] Bilge Mutlu and Jodi Forlizzi. 2008. Robots in Organizations: The Role of Workflow, Social, and Environmental Factors in Human-Robot Interaction. In Human-Robot Interaction (HRI), 2008 3rd ACM/IEEE International Conference on, 287-294.

[40] Bilge Mutlu, Steven Osman, Jodi Forlizzi, Jessica Hodgins, and Sara Kiesler. 2006. Task Structure and User Attributes as Elements of Human-Robot Interaction Design. In Robot and Human Interactive Communication, 2006. ROMAN 2006. The 15th IEEE International Symposium on, 74-79.

[41] Hiroshi Nakajima, Ryota Yamada, Scott Brave, Yasunori Morishima, Clifford Nass, and Shigeyasu Kawaji. 2003. The Functionality of Human-Machine Collaboration Systems-Mind Model and Social Behavior. In Systems, Man and Cybernetics, 2003. IEEE International Conference on, 2381-2387.

[42] Tatsuya Nomura, Takuya Shintani, Kazuki Fujii, and Kazumasa Hokabe. 2007. Experimental Investigation of Relationships between Anxiety, Negative Attitudes, and Allowable Distance of Robots. In Proceedings of the 2nd IASTED International Conference on Human-Computer Interaction, Chamonix, France. ACTA Press, 13-18.

[43] Tatsuya Nomura, Tomohiro Suzuki, Takayuki Kanda, and Kensuke Kato. 2006. Measurement of negative attitudes toward robots. Interact. Stud. 7, 3 (December 2006), 437-454.

[44] Kristin E. Oleson, D. R. Billings, Vivien Kocsis, Jessie YC Chen, and Peter A. Hancock. 2011. Antecedents of Trust in Human-Robot Collaborations. In Cognitive Methods in Situation Awareness and Decision Support (CogSIMA), 2011 IEEE First International Multi-Disciplinary Conference on, 175-178.

[45] Nadia Olivero and Peter Lunt. 2004. Privacy versus willingness to disclose in ecommerce exchanges: The effect of risk awareness on the relative role of trust and control. F. Econ. Psychol. 25, 2 (April 2004), 243-262. DOI:https://doi.org/10.1016/S0167-4870(02)00172-1

[46] Clark Patrick and Kim Bhasin. 2017. Amazon's Robot War Is Spreading. (April 5, 2017). Retrieved Jan. 3, 2018 from https://www.bloomberg.com/news/articles/2017-04-05/robots-enlist-humansto-win-the-warehouse-war-amazon-started

[47] K. W. Phillips, G. B. Northcraft, and M. A. Neale. 2006. Surface-level diversity and decision-making in groups: When does deep-level similarity help? Group Process. Intergroup Relat. 9, 4 (October 2006), 467-482. DOI:https://doi.org/10.1177/1368430206067557 
[48] Mohammed Owais Qureshi and Rumaiya Sajjad Syed. 2014. The impact of robotics on employment and motivation of employees in the service sector, with special reference to health care. Saf. Health Work 5, 4 (December 2014), 198-202. DOI:https://doi.org/10.1016/j.shaw.2014.07.003

[49] Lionel P. Robert. 2013. A Multi-level Analysis of the Impact of Shared Leadership in Diverse Virtual Teams. In Proceedings of the 2013 Conference on Computer-supported Cooperative Work, 363-374.

[50] Lionel P. Robert, Alan R. Denis, and Yu-Ting Caisy Hung. 2009. Individual swift trust and knowledge-based trust in face-to-face and virtual team members. 7. Manag. Inf. Syst. 26, 2 (2009), 241-279.

[51] Lionel P. Robert and Tracy Ann Sykes. 2016. Extending the concept of control beliefs: Integrating the role of advice networks. Inf. Syst. Res. 28, 1 (2016), 8496.

[52] Paul Robinette, Wenchen Li, Robert Allen, Ayanna M. Howard, and Alan R. Wagner. 2016. Overtrust of Robots in Emergency Evacuation Scenarios. In Human-Robot Interaction (HRI), 2016 11th ACM/IEEE International Conference on, 101-108.

[53] Joshua M. Sacco, Christine R. Scheu, Ann Marie Ryan, and Neal Schmitt. 2003. An investigation of race and sex similarity effects in interviews: A multilevel approach to relational demography. 7. Appl. Psychol. 88, 5 (2003), 852.

[54] Maha Salem, Gabriella Lakatos, Farshid Amirabdollahian, and Kerstin Dautenhahn. 2015. Would You Trust a (Faulty) Robot?: Effects of Error, Task Type and Personality on Human-Robot Cooperation and Trust. In $10^{\text {th }}$ ACM/IEEE International Conference on Human-Robot Interaction, 141-148.

[55] Lauren E. Scissors, Alastair J. Gill, Kathleen Geraghty, and Darren Gergle. 2009. In CMC We Trust: The Role of Similarity. In Proceedings of the SIGCHI Conference on Human Factors in Computing Systems, 527-536.

[56] Sam Shead. 2017. Amazon Now has 45,000 Robots in its Warehouses. (Jan. 3 , 2017). Retrieved Jan. 3, 2018 from http://www.businessinsider.com/amazonsrobot-army-has-grown-by-50-2017-1

[57] Ramadhar Singh, Duane T. Wegener, Krithiga Sankaran, Smita Singh, Patrick K. F. Lin, Mellissa Xuemei Seow, Jocelyn Shu Qing Teng, and Sudderuddin Shuli. 2015. On the importance of trust in interpersonal attraction from attitude similarity. 7. Soc. Pers. Relatsh. 32, 6 (2015), 829-850.

[58] Harold L. Sirkin, Michael Zinser, and Justin Rose. 2015. Industries and Economies Leading the Robotics Revolution. (Sept. 23, 2015). Retrieved Jan. 3, 2018 from https://www.bcgperspectives.com/content/articles/leanmanufacturing-innovation-industries-economies-leading-robotics-revolution/

[59] James Surowiecki. 2017. Chill: Robots Won't Take All Our Jobs. (Aug. 16, 2017) Retrieved Jan. 3, 2018 from https://www.wired.com/2017/08/robots-will-nottake-your-job/

[60] Leila Takayama, Wendy Ju, and Clifford Nass. 2008. Beyond Dirty, Dangerous and Dull: What Everyday People Think Robots Should Do. In Proceedings of the 3rd ACM/IEEE International Conference on Human-Robot Interaction, 25-32.
[61] Adriana Tapus and Maja J. Mataric. 2008. Socially Assistive Robots: The Link between Personality, Empathy, Physiological Signals, and Task Performance. In AAAI Spring Symposium: Emotion, Personality, and Social Behavior, 133-140.

[62] Benedict Tay, Younbo Jung, and Taezoon Park. 2014. When stereotypes meet robots: the double-edge sword of robot gender and personality in humanrobot interaction. Comput. Hum. Behav. 38, (2014), 75-84.

[63] Rolf Van Dick, Daan Van Knippenberg, Silvia Hägele, Yves RF Guillaume, and Felix C. Brodbeck. 2008. Group diversity and group identification: The moderating role of diversity beliefs. Hum. Relat. 61, 10 (2008), 1463-1492.

[64] Viswanath Venkatesh and Fred D. Davis. 2000. A theoretical extension of the technology acceptance model: Four longitudinal field studies. Manag. Sci. 46, 2 (2000), 186-204.

[65] Ewart de Visser and Raja Parasuraman. 2011. Adaptive aiding of human-robot teaming effects of imperfect automation on performance, trust, and workload. f. Cogn. Eng. Decis. Mak. 5, 2 (2011), 209-231.

[66] MaryJo Webster. 2014. Could a Robot Do Your Job? (Oct. 28, 2014). Retrieved

3 from https://www.usatoday.com/story/news/nation/2014/10/28/low-skill-workersface-mechanization-challenge/16392981/

[67] Sarah Woods, Kerstin Dautenhahn, Christina Kaouri, R. Boekhorst, and Kheng Lee Koay. 2005. Is This Robot Like Me? Links between Human and Robot Personality Traits. In Humanoid Robots, 2005 5th IEEE-RAS International Conference on, 375-380.

[68] Sarah Woods, Kerstin Dautenhahn, Christina Kaouri, René te Boekhorst, Kheng Lee Koay, and Michael L. Walters. 2007. Are robots like people?: Relationships between participant and robot personality traits in human-robot interaction studies. Interact. Stud. 8, 2 (2007), 281-305.

[69] Holly A. Yanco and Jill L. Drury. 2004. Classifying human-robot interaction: An updated taxonomy. In SMC (3), 2841-2846.

[70] Sangseok You and Lionel P. Robert. forthcoming. Emotional Attachment, Performance, and Viability in Teams Collaborating with Embodied Physical Action (EPA) Robots. 7. Assoc. Inf. Syst. (forthcoming).

[71] Sangseok You and Lionel P. Robert. 2017. Teaming up with robots: An IMOI (inputs-mediators-outputs-inputs) framework of human-robot teamwork. Int. 7 . Robot. Eng. 2, 003 (2017).

[72] Sangseok You, Teng Ye, and Lionel Robert. 2017. Team Potency and Ethnic Diversity in Embodied Physical Action (EPA) Robot-Supported Dyadic Teams. In Proceedings of the 38th International Conference on Information Systems (ICIS 2017). Seoul, South Korea. December 10-13. AIS.

[73] Mary E. Zellmer-Bruhn, Mary M. Maloney, Anita D. Bhappu, and Rommel Bombie Salvador. 2008. When and how do differences matter? An exploration of perceived similarity in teams. Organ. Behav. Hum. Decis. Process. 107, 1 (2008), 41-59. 